\title{
Estudio de recepción de una campaña audiovisual sobre prevención del consumo de sustancias psicoactivas ${ }^{1 .}$
}

\author{
Reception study an audiovisual campaign on prevention of psychoactive \\ substances.
}

Dina Sofía Gualdrón Alvarez ${ }^{2}$

Julián David Vélez Carvajal ${ }^{3}$

Gualdrón A. Dina S, Vélez C. Julián D. miradas N¹3 - 2015. ISSN: 0122 994X Págs 124 - 138

Recepción: Mayo 14 de 2015

Aprobación: Octubre 8 de 2015

Publicación: Diciembre 17 de 2015

\section{Resumen}

El presente artículo hace un recorrido por las televidencias, recepción, audiencias y mediaciones, sustentadas bajo el pensamiento del autor mexicano Guillermo Orozco Gómez, este autor reconoce la presencia de múltiples mediaciones (macro y micro) que pueden llegar a ser puntos de referencia en el planteamiento de procesos de comunicación educativa para los niños y adolescentes que encuentran en las campañas de prevención una oportunidad para reevaluar comportamientos, inquietudes y decisiones frente a la problemática del consumo de sustancias psicoactivas en la Institución Educativa Distrital Manuela Ayala de Gaitán. La propuesta tuvo un enfoque comprensivo - interpretativo de las mediaciones y las recepciones en los estudiantes con el fin de visibilizar, inicialmente, las mediaciones y establecer relación entre lo audiovisual y la recepción de los mensajes de prevención de consumo de sustancias psicoactivas; a la vez permitió la posibilidad de reconstruir los sentidos que permanecen implícitos en las acciones y los discursos de los sujetos. La técnica de recolección de la información fue el grupo focal adaptado debido a la edad de los estudiantes que oscila entre los 10 y 12 años, donde se plantearon dinámicas de representación gráfica, teatral y construcción de comics. El análisis de los datos se llevó a cabo mediante el análisis/síntesis que permitió relacionar de manera implícita y explicita la teoría y los datos. Esta investigación reconoce la presencia de

1 El presente artículo surge a partir de la investigación, Recepción de una campaña audiovisual sobre prevención del consumo de sustancias psicoactivas en estudiantes de grado $5^{\circ} \mathrm{jt}$ (Jornada Tarde), institución educativa distrital Manuela Ayala de Gaitán (Bogotá D.C).

2 Licenciada en Educación Básica, especialista en Comunicación educativa, Magister en Comunicación Educativa, Universidad Tecnológica de Pereira. Docente de básica primaria de la Secretaría de Educación Distrital de Bogotá, acreedora de Incentivos 2014-2015. sofias@utp.edu.co

3 Licenciado en Etnoeducación y Desarrollo Comunitario, Magister en Comunicación Educativa, Candidato a Doctor en Ciencias de la Educación, área Pensamiento Educativo y Comunicación. Docente de la Universidad Tecnológica de Pereira.jvel@utp.edu.co 
múltiples mediaciones (macro y micro), que pueden llegar a ser puntos de referencia en el planteamiento de procesos de comunicación educativa para los niños $\mathrm{y}$ adolescentes que encuentran en las campañas de prevención una oportunidad para reevaluar comportamientos, inquietudes $y$ decisiones frente a la problemática del consumo de sustancias psicoactivas. Se pudo establecer que lo emocional y lo contextual, hacen parte fundamental del proceso de recepción de los niños. Asimismo, familia, escuela y televisión se constituyen en instituciones que comparten la formación de los niños.

Palabras Clave: Recepción, Audiencias, Micromediaciones y Macromediaciones.

\section{Abstract}

The following article goes around viewers, reception, audience and mediations sustained under the thought of the Mexican author Guillermo Orozco Gómez, who recognizes the presence of multiple mediations (macro and micro), that can become as points of reference in the recognition of interaction processes with television screen process for children and teenagers who are related with the prevention of consuming psychoactive substances campaigns in the official institution Manuela Ayala de Gaitan in Bogota. The proposal had a comprehensive-interpretive approach of student's mediations and receptions, in order to visualize, initially mediations and stablish the relation between the audiovisual and messages reception about psychoactive substances consumption prevention; simultaneously it allowed the possibility of reconstructing the senses that remain implicit in the actions and subjects' speech. The technique used for information compilation was the focal group, due to the students' age, which ranges between 10 and 12 years old, where were planned dynamic of graphical and theatrical representation, and construction of comics. The analysis of the information was carried out by analysis/synthesis processes developed by the investigation group of master in educational communication from Universidad Tecnológica de Pereira that allowed relating in an implicit and explicit way, the theory and the information. This research recognizes the presence of multiple mediations (macro and micro), that might be as a point of reference in the proposal of educational communication process for children and teenagers who find in the campaigns of prevention an opportunity to re-evaluate behaviors, worries and decisions facing psychoactive substances consumption problematic.

Key Words: Reception, audiences, Micromediations, Macromediations

\section{Introducción}

El análisis que se presenta en el artículo parte de la revisión de las mediaciones que se pueden llegar a dar entre el sujeto y una campaña audiovisual sobre prevención de sustancias psicoactivas en la Institución Educativa Distrital Manuela Ayala de Gaitán de la ciudad de Bogotá. Igualmente, se pretende cuestionar la forma como los jóvenes desarrollan los procesos de recepción, interpretación, resignificación y adopción de los referentes de la televisión (Orozco, 2001), y, muy especialmente, a los mensajes publicitarios, ya que en el contexto escolar no se prepara a las audiencias infantiles y juveniles para hacer procesos activos de interpelación con la pantalla. Se desconoce la complejidad de los procesos de recepción y apropiación de los mensajes que va mucho más allá de la pantalla, aún poco estudiada y comprendida en el ámbito de la comunicación - educación, lo 
poco que se conoce es extrapolado de otros entornos culturales.

En lo que concierne a la relación entre la recepción y las campañas publicitarias sobre el consumo de sustancias psicoactivas, es necesario relacionar la mediación y la eficacia de las campañas, para abordar la recepción como patrón de análisis que permite no solo el llevar a cabo un proceso investigativo, sino evidenciar la manera como los estudiantes pueden llegar a ser audiencias activas en cada uno de los contextos televisivos observados.

La investigación tuvo un enfoque comprensivo de las mediaciones y las recepciones en los estudiantes con el fin de visibilizar inicialmente las mediaciones y establecer relaciones entre lo audiovisual y la recepción de los mensajes de prevención de consumo de sustancias psicoactivas, lo cual permite reconstruir los sentidos que permanecen implícitos en las acciones y los discursos de los sujetos.

\section{Estudiantes- Audiencias}

Se contó con una población objeto de estudio de 20 estudiantes que oscilan entre los 10 y 12 años de edad, etapa de descubrimiento y exploración de culturas juveniles; además, atraviesan una etapa de transición entre la infancia y la pubertad, en el ámbito psicosocial empiezan a comportarse de manera distinta y a expresar intereses particulares. En lo emocional tanto para los niños como para las niñas el grupo de amigos adquiere gran relevancia, es aquí donde se hace necesaria una orientación sobre el consumo para evitar la entrada a este universo.

Como instrumento, se utilizó el grupo focal adaptado, conservando la premisa de dicho instrumento como una técnica de recolección de datos mediante una entrevista grupal semiestructurada, la cual gira alrededor de una temática propuesta por el investigador.

La adaptación se hace necesaria debido a la edad de los estudiantes que oscila entre los 10 y 12 años, planteando dinámicas a partir de las campañas audiovisuales observadas en el aula.

Se aplicaron 4 grupos focales, el primero basado en la representación gráfica y teatral de comerciales con el fin tener una visión holística de aquello que están observando los niños en su cotidianeidad. Teniendo como referente la teoría de Orozco frente a la importancia de entender la imagen y la interacción que se debe tener con la misma para entender, conocer y disfrutar a través de ella.

El segundo se aplica luego de observar la campaña sobre prevención de consumo de sustancias psicoactivas: "Campaña mundial sobre prevención de consumo de sustancias psicoactivas 2011", aquí se sugiere la elaboración de una historieta donde se refleje la idea principal de la campaña observada. Ya que estas (comics o historietas) ofrecen una información visual clara y representativa de acciones, ofreciendo secuencias completas con todo detalle y aportando una gran cantidad y diversidad de contenidos. Además, permiten presentar contenidos en un formato atractivo y motivador, pudiendo ser orientados a un espectro amplio de edades, para el caso la edad escolar.

En el tercer y cuarto grupo focal respectivamente, se trabajó la entrevista grupal semiestructurada. Para la aplicación de la misma se diseñaron dos guías para ser desarrolladas en grupo, donde se plantearon tres preguntas (en cada guía) relacionadas con la categoría de micromediaciones (guía 3) y macromediaciones (guía 4), en ésta a la vez se planteó la representación teatral como complemento a las respuestas dadas. 


\section{Expresiones sin palabras...}

Para la implementación de los grupos focales, se hizo uso de materiales como: papel, lápices de colores y videograbadoras con el propósito de que los estudiantes elaboraran las historietas e hicieran las representaciones teatrales. Para ello, se puso en condición de visionado la campaña mundial sobre prevención de consumo de sustancias psicoactivas del año 2011.

La aplicación del instrumento se realizó en lapsos de tiempo de una hora durante 4 días consecutivos; inicialmente, se hizo una socialización del propósito del proyecto, momento en que surgió la pregunta por el termino sustancias psicoactivas (SPA), lo cual se explicó con el sinónimo más conocido por los participantes como lo son las drogas; seguidamente, se recalcó en la importancia de la disponibilidad esperada por parte de cada uno de los participantes, para el caso concreto de la primera guía de trabajo, se organizaron 4 grupos, 2 de 4 estudiantes y 2 de 5 respectivamente.

Se entregó una guía por grupo, luego se explicó la manera como se debían desarrollar cada una de las actividades planteadas en las guías. Para citar un ejemplo, en el grupo focal 1, el primer punto consistía en hacer una representación gráfica del comercial de televisión que más les llame la atención a cada uno de los participantes, para ello se contaba con un tiempo de $15^{\prime}$ minutos, para luego, en el segundo, hacer una representación teatral por grupo donde se socializara uno de los comerciales representados.

Explicada la guía, cada grupo inició su trabajo, llama la atención la manera como surgen conversaciones en los grupos alrededor de lo propuesto, pues antes de dibujar, comparten con los demás ccompañeros de grupo lo que les gustaría representar, algunos hablan de fútbol, otros de programas de televisión en el canal Disney, y luego cada uno plasma sus intereses.

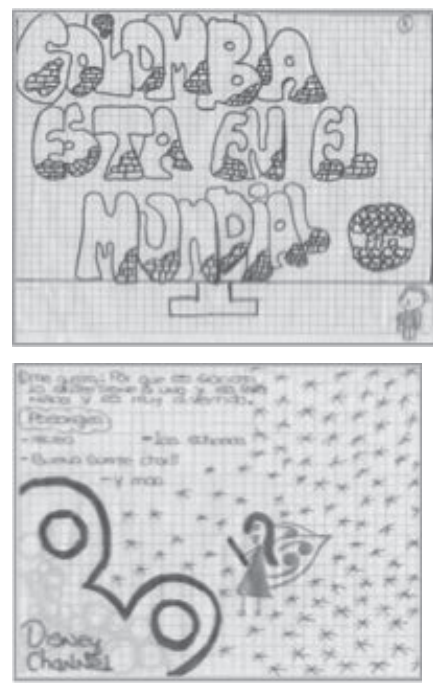

Graficas 1y 2 : Representaciones gráficas, Mi comercial favorito

Así, en cada uno de los encuentros se explicó inicialmente la dinámica a realizar y los tiempos con los cuales se disponía para tal fin. Luego de que se observó la campaña se dio la instrucción de realizar un comic por grupo (se organizaron 5 grupos de 4 participantes cada uno) resaltando la idea principal de la campaña (grupo focal 2). A continuación se presenta uno de los comics construidos luego de la proyección de la campaña audiovisual:

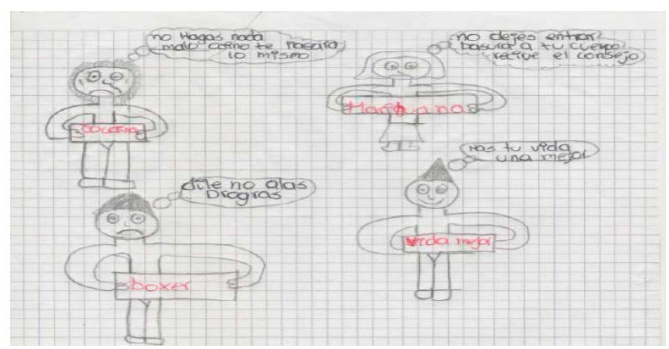

Gráfica 3: Comic, idea principal de la campaña sobre prevención del consumo de SPA 
Durante la aplicación de dicho grupo focal, se observa a los grupos hablando acerca de lo que vieron en la campaña, la situación de cada personaje y cómo se vio afectada la salud de cada uno de ellos a causa de las sustancias psicoactivas.

En el comic anterior se tienen presentes los personajes de la campaña y la idea principal que resalta es la de: "Fue la peor decisión que tome", "Me odio por haberme hecho eso" y "haz tu vida mejor"; la cual surge luego de la observación de la campaña publicitaria.

Posteriormente, se aplican los grupos focales 3 y 4 , donde se realizan entrevistas semiestructuradas a los participantes y se sugiere la representación teatral de las ideas resultantes. A continuación se toma un pantallazo de una de las representaciones donde se presentó el rol de la televisión frente a la prevención del consumo de sustancias psicoactivas.

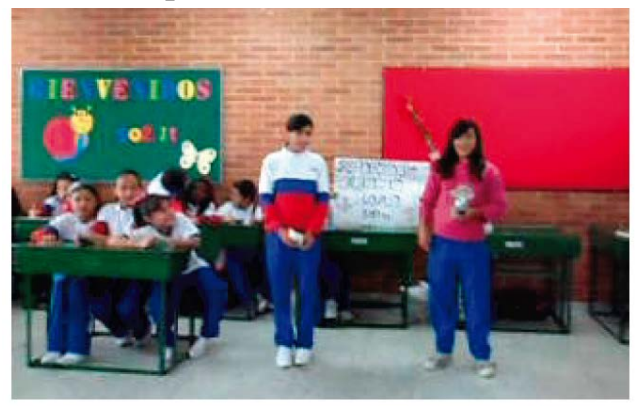

Grafica 4: Representación teatral, función de la televisión en la prevención del consumo de SPA

Descripción general de la imagen: el grupo se integra por 5 estudiantes que representan la función de la televisión en la prevención del consumo de sustancias psicoactivas. A continuación, se describe el proceso realizado:

"LUGAR: set de información, se hace una representación de un programa de televisión sobre la temática de prevención de consumo de sustancias psicoactivas (SPA), se observa de fondo una cartelera con la frase "reportaje última hora, 7:00 pm"
PRESENTADORA 1: "(utilizando un micrófono) Muy Buenas tardes, mi nombre es Ginna Alejandra Romero Lara, y venimos aaa... hoy vengo a reportar sobre las campañas que se realizan contra el consumo de drogas en niños y adolescentes...ehh venimos con mi compañera Mayerly"

PRESENTADORA 2: “(recibe el micrófono) aquí en el Colegio Manuela Ayala de Gaitán en la localidad de Engativá...eee aquí le paso a mi compañera.

PRESENTADORA 3: “(recibe el micrófono) hay varias campañas para la prevención de drogas en niños y adolescentes, son varios nombres de drogas...mi compañera...Ana María..."

PRESENTADORA 4: "esos nombres son crack, tiner, cocaína, marihuana y muchos más, vamos con Laura..."

PRESENTADORA 5: "mis muchachos y muchachas, también juntos trataremos de llevar una vida sana para poder salir adelante...gracias".

(Se escuchan aplausos)."

Lo anterior permitió realizar el proceso de análisis/síntesis de la información recolectada, donde se observa en cada imagen y representación teatral un sinnúmero de emociones, sentimientos y sobre todo un deseo por la prevención del consumo de SPA y cada una de las situaciones que de allí se derivan.

\section{Moliendo, afinamos el dato.}

A partir de los diferentes datos compuestos por representaciones gráficas, teatrales, comics y entrevistas es necesario realizar el proceso de análisis/síntesis del dato, por lo tanto, la información obtenida en cada uno de los grupos focales se sometió a dicho procedimiento.

Para cada grupo focal, se emplearon diferentes plantillas que facilitaron la lectura y descripción de cada uno de los dibujos, videos y entrevistas, lo anterior tomando como referente los índices relacionados con los objetivos y la pregunta de investigación y las posibles categorías asociadas. 
Luego del proceso realizado se procede a identificar palabras $y / 0$ frases resultantes en cada grupo focal relacionadas con la pregunta de investigación, donde se da cuenta del número de veces que aparece cada una de ellas:

\begin{tabular}{|l|c|l|l|}
\hline \multicolumn{4}{|c|}{ RECURRENCLA O SIMLITUD DE LAS PALABRAS } \\
\hline Colombia & 2 & Sonrisa & 2 \\
\hline Mundial & 1 & Canal Disney & 5 \\
\hline FIFA & 1 & Divertico & 2 \\
\hline Futbol & 5 & Hada madrina & 1 \\
\hline Escado equipo de futbol & 3 & Barita mágica & 1 \\
\hline Ojos grandes & 2 & Gusto & 1 \\
\hline Pepa cerdita & 6 & Atención & 1 \\
\hline Lodo & 1 & Televisor & 1 \\
\hline Mamá & 6 & Habitación & 1 \\
\hline Papá & 6 & & \\
\hline Hija & 6 & & \\
\hline Hermano & 6 & & \\
\hline Franja familiar & 4 & & \\
\hline Mesa noche & 1 & & \\
\hline
\end{tabular}

Tabla 1: Grupo focal 1: Mi comercial favorito

Parte1: Representaciones graficas

\section{Proceso de reagrupación.}

Después de tener los conjuntos se pensó en un término, palabra o concepto que los reuniera. Para dicha agrupación se tuvieron en cuenta la totalidad de los esquemas expuestos anteriormente. A la vez, se asociaron las categorías planteadas por el autor, presentes en el marco teórico, las cuales se ubican en el centro del esquema. He aquí una muestra de las reagrupaciones.

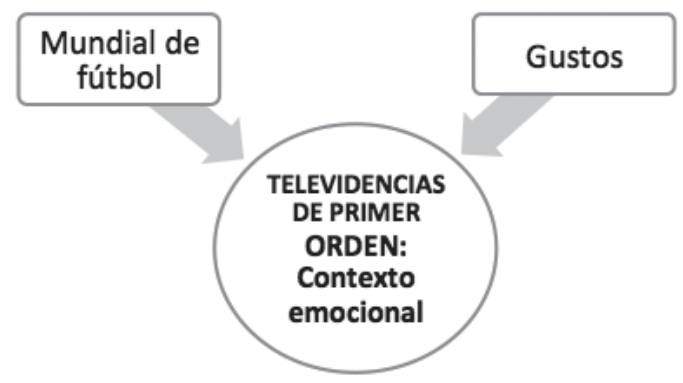

Esquema 1: Categoría micromediaciones:

Televidencias de primer orden, contexto emocional

En el esquema arriba mencionado, se evidencian las mediaciones que surgen debido al contexto en el que se encuentran los estudiantes y a la vez, las emociones que se pueden llegar a construir debido a los gustos de cada uno y el evento de fútbol más importante del mundo.

\section{Hacia un pensamiento circular.}

Después de haber realizado cada una de las agrupaciones surgen los diagramas que permitieron reagrupar cada una de las categorías resultantes, concentrando el dato, lo cual servirá como elemento fundamental en la posterior discusión con el autor, para lo cual son utilizados los diagramas de Venn.

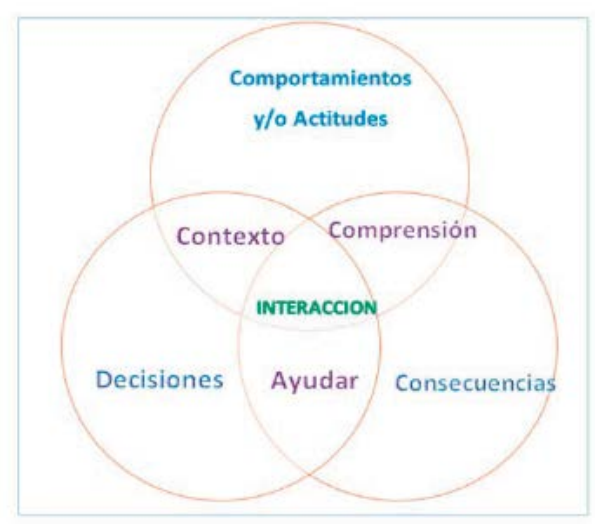

Esquema 2: Micromediaciones: televidencias de segundo orden, interacción

Aquí surgen los diagramas que compilan, de cierta manera, todo un análisis/síntesis del dato; inicialmente, se visualizan las micromediaciones que surgen del proceso inicial de recepción y primer contacto con la campaña audiovisual sobre prevención del consumo de sustancias psicoactivas.

En el esquema 4, se compilan un grupo de micromediaciones relacionadas con la interacción como televidencia de segundo orden, que se produce fuera del espacio de interacción con la televisión y en un tiempo distinto, es decir, antes o después de la exposición televisiva. 
Luego, se introducen las macromediaciones relacionadas con las instituciones (ver esquema 4.1):

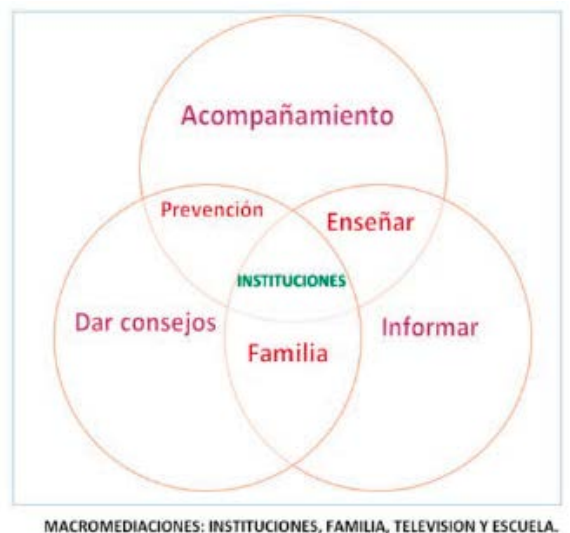

Esquema 4.1: Macromediaciones: instituciones, familia, televisión y escuela

Aquí se observa una panorámica de la macro mediación institución, que permite posicionar a las instituciones familia y escuela como primarias en el proceso de socialización; paralelamente, en la parte exterior del diagrama se exponen las funciones más relevantes en la prevención del consumo de SPA.

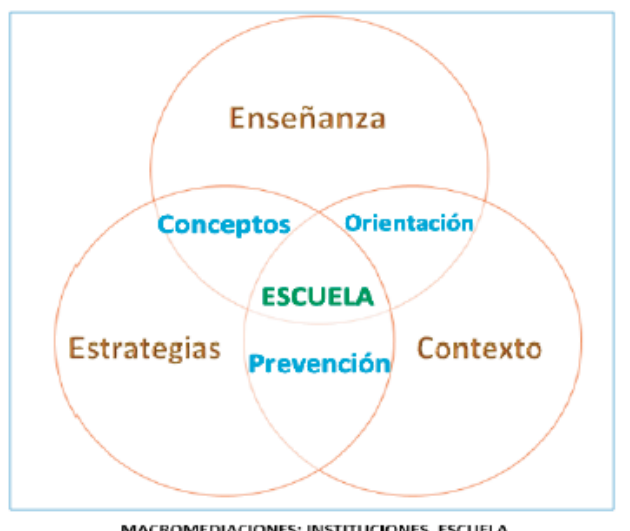

Esquema 4.2: Macromediaciones: instituciones, escuela

Al articular el diagrama 4.1 y 4.2 es reiterativa la presencia de la escuela como institución mediadora en los procesos de recepción de los niños, para lo cual Orozco propone la tarea de "televidenciar", es decir, hacer evidente lo que no lo es, tanto de la televisión como de las interacciones o televidencias que con ella entablan sus audiencias.

Con esto no se trata de reprimir, prohibir o criticar, sino de propiciar en la escuela espacios para el análisis y la reflexión de los sujetos - audiencia orientando el aprendizaje televisivo. Lo anterior permitiría cuestionamientos más allá de tener una mirada crítica respecto a los contenidos televisivos; el fin último es la construcción de una sociedad más justa y democrática; constituida por sujetos capaces de una visión crítica y transformadora, no solo de la televisión, sino de todos aquellos aspectos que integran la vida social.

En este orden de ideas surge una agrupación final que da cuenta global de todo el proceso de análisis/síntesis de la información, con el fin de responder los objetivos de la investigación y la pregunta planteada.

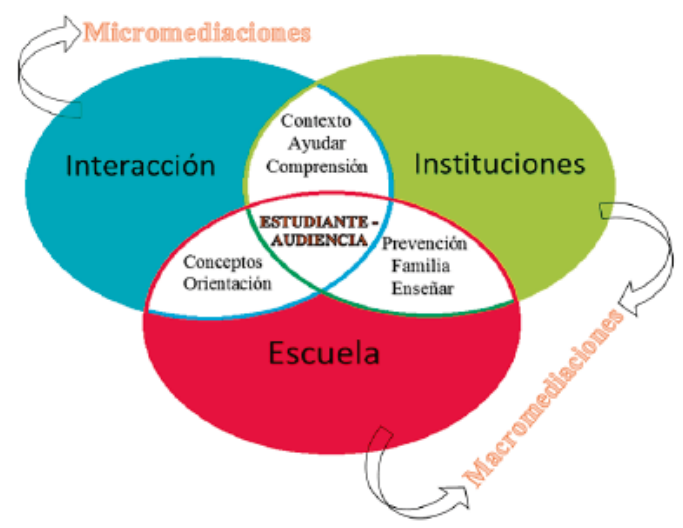

Esquema 3: Micromediaciones y macromediaciones visibilizadas en el proceso de recepción

Inicialmente, se visualiza la interacción como una de las micromediaciones fuertes que contienen a la vez el contexto, el ayudar y la comprensión (esto haciendo referencia a que la campaña audiovisual 
respondía a la prevención de consumo de sustancias psicoactivas), se puede extraer de aquí la relevancia que tiene para los menores participantes en dicha investigación el hecho de hacer parte de un contexto donde el consumo de sustancias psicoactivas es cada vez más cercano, he aquí una representación particular de uno de los grupos:

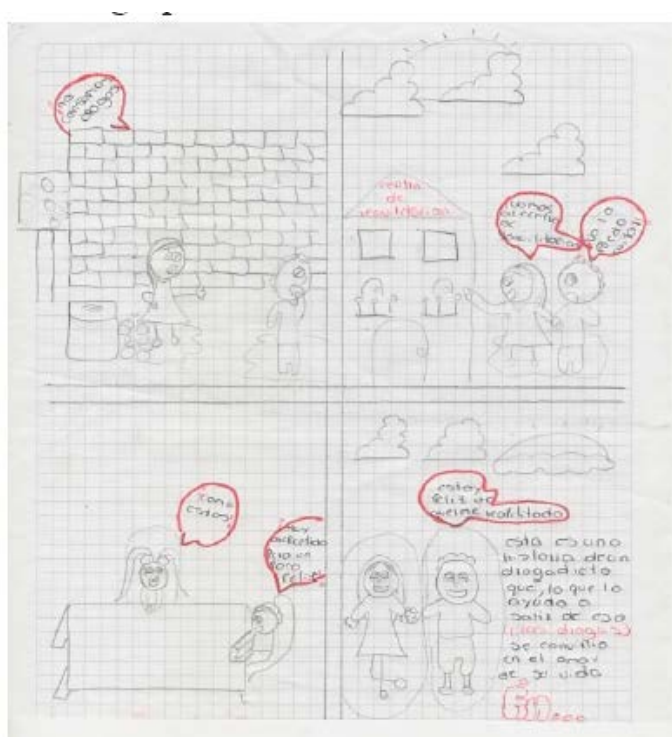

Tira cómica Grupo 3

Aunque pareciera que las diferentes problemáticas del entorno próximo pasaran desapercibidas para los menores, ellos muestran una gran preocupación por lo que sucede a su alrededor y a la vez proponen alternativas para prevenir determinadas situaciones.

Desde ahí que la progresiva mediatización de la vida y la socialización está incitando diversos cambios y conflictos en las dinámicas familiares y escolares, y Orozco identifica que uno de ellos reside en los "resultados", es decir, lo que se espera de cada institución social; los jóvenes con frecuencia hacen comparaciones entre los mensajes emitidos por cada institución, con la información que adquieren de los medios, y esto a su vez genera otro cuestionamiento que transforma "el proceso" de cada institución al poner en tela de juicio sus funciones.

Pese a las mediaciones (macro y micro), los mensajes televisivos en muchos casos logran imponerse porque son capaces de ir más allá del "acto de ver televisión", al volverse insumos y referentes de las relaciones sociales. Este proceso preocupa a Orozco porque sucede de forma tan implícita que muchas veces pasa desapercibido, de ahí que sea necesario rescatar cuáles son "los recursos" con los que debe contar cada institución social para generar resistencias (mediaciones), más certeras que permitan no sólo mantener sus funciones sociales (preservar los valores y educar), sino también usar las posibilidades emitidas por la televisión como un insumo para lograr esos mismos fines.

De manera que el receptor, como agente activo, no sólo "negocia" los significados televisivos sino que también los traspone a otras situaciones sociales próximas, esto le permite "re-apropiar y resignificar" lo observado en la televisión a favor de los fines personales y en relación con los significados comunes que comparten los contextos de interpretación a los que el receptor pertenece (familia, escuela, amigos, barrio, iglesia y ahora las redes sociales disponibles en internet).

Así que en este orden de ideas la responsabilidad en la formación de nuestros estudiantes es compartida por las instituciones familia- escuela, puesto que el estudiante como televidente inicia un proceso de resistencia (mediaciones), frente a la campaña audiovisual sobre prevención de consumo de sustancias psicoactivas, a la vez se dan unas interacciones entre lo que ve y vive; paralelamente cada una de las instituciones (familia-escuela), debe ejercer determinadas funciones, esto se 
sustenta teniendo en cuenta las respuestas dadas a las siguientes preguntas:

\section{Grupo Focal 3 y 4}

¿Cuál es la función de la familia en la prevención del consumo de sustancias psicoactivas?

\section{Respuestas}

1: "que la prevención de la familia es que lo consejen para que no se vuelva a adicto”

2: “ ayudarse entre familia dándose consejos y tener presente sobre lo malo que son las sustancias psicoactivas"

3: "la función principal de la familia es aconsejarlos para que no caigan en la tentación de las drogas"

4: “deben darle un consejo a sus hijos y no solo eso sino a enseñarles que no lo deben consumir ni recibirle a nadie nada"

Los estudiantes esperan de la familia como institución, un espacio donde se den consejos para prevenir el consumo de sustancias psicoactivas, puesto que éste es visto como una "tentación" que perciben como negativa en su contexto.

Paralelamente, al indagar por la función de la institución educativa, responden:

¿...Cuál es la función de la institución educativa en la prevención del consumo de sustancias psicoactivas?

\section{Respuestas:}

1: "prevenir sustancias alcohólicas en el colegio escuchar temas que nos ayuden al no ser alcohólico"

2: "platicar con los estudiantes sobre las sustancias psicoactivas para que no se vuelvan consumidores de sustancias psicoactivas"

3: "el colegio nos deben dar proyectos o talleres para evitar el consumo de sustancias ecoactivas (psicoactivas)"

4: "censurar al estudiante que lo consume adentro o fuera del colegio o depende de la decisión $q$ tome el rector y el coordinador o mandarlo a una correccional de menores"
5: "hacer campañas contra el consumo de drogas en niños o adolescentes de la institución”"

Aquí se observa una necesidad sentida por los estudiantes, frente determinadas estrategias para la prevención del consumo de SPA, tales como: talleres, charlas, campañas y proyectos.

Los estudiantes de la Institución Educativa Distrital Manuela Ayala de Gaitán, manifiestan una preocupación por lo que está sucediendo en el entorno próximo, no son ajenos a las problemáticas y se generan propuestas frente a la prevención del consumo de SPA. Se visualiza una necesidad marcada de apoyo familiar y escolar; lo que afirma que si bien las pantallas juegan un papel importante en la cotidianeidad de nuestros estudiantes, el proceso de recepción es retroalimentado en las aulas y cada una de las familias; siendo éstas dos de las macromediaciones fuertes presentes en los procesos de recepción de la campaña audiovisual sobre prevención de consumo de sustancias psicoactivas.

Por consiguiente, en el proceso de recepción se marcan inicialmente unas micromediaciones que responden a la interacción con el mensaje de la campaña audiovisual, lo cual indica que se está dando un proceso de comunicación activo entre los estudiantes en el rol de televidentes.

Progresivamente, se afianza la macromediación de las instituciones (familia- escuela), puesto que la temática de la campaña toca un aspecto bastante susceptible en los espacios enunciados, los estudiantes se encuentran mediados por éstas instituciones, ya que se incluyen en su cotidianidad y es aquí donde se dan una serie de ritualidades que hacen parte del proceso de recepción.

Como lo plantea Orozco, hay una construcción de los significados, en la 
medida que antes de observar la campaña audiovisual, existen unos preconceptos sobre la temática y luego se avanza a la encodificación. Paralelamente, hay una proposición de significados que se evidencian mediante la representación gráfica y teatral de la idea principal de la campaña, se proponen nuevos significados lo cual indica que se está entendiendo una idea; se está extrayendo del código el conjunto de significados que conforman posiciones de lecturas específicas.

De manera que se hace evidente "la negociación" mediante la cual se aceptan o rechazan los mensajes televisivos. Los estudiantes son capaces de crear y sustentar situaciones que complementan de cierta manera, lo visualizado en la campaña audiovisual.

Lo anterior indica una reapropiación en los contenidos, a través de una secuencia que arranca con la atención, pasa por la comprensión, la selección, la valoración de lo percibido, su almacenamiento e integración con informaciones anteriores $y$, finalmente, se realiza una apropiación y una producción de sentido. Cabe aclarar que dicha secuencia se puede dar de diferentes maneras y a ritmos diferentes.

Efectivamente, la razón primordial es que la secuencia de actividades conlleva a una serie de agrupaciones de contenido, en este caso entre la información dada por la pantalla y, por lo tanto, exterior al sujeto y la información previamente asimilada por el televidente.

\section{Visibilizando mediaciones para una Educación-comunicación en recepción}

La presente investigación reconoce la presencia de múltiples mediaciones (macro y micro), que pueden llegar a ser puntos de referencia en el planteamiento de procesos de Comunicación Educativa para los niños y adolescentes que encuentran en las campañas de prevención una oportunidad para reevaluar comportamientos, inquietudes y decisiones frente a la problemática del consumo de sustancias psicoactivas.

Paralelamente, se logró reconocer un grupo de mediaciones que se encuentran directamente relacionadas con las interacciones de los niños en el contexto y las instituciones (familia - escuela). Lo que deja ver la influencia de diferentes contextos (emocional y espacial), como mediación sustancial al momento de observar una campaña sobre prevención de consumo de sustancias psicoactivas.

De otro lado, la macromediación institucional (familia-escuela), permite evidenciar la necesidad de orientaciones concretas para la toma de decisiones por parte de las instituciones enunciadas. Es aquí donde se plantea una pedagogía comunicativa, educar para los medios y esto solo se logra cuando se tienen claras las múltiples mediaciones que experimenta la audiencia al observar determinada campaña o contenido televisivo.

El planteamiento anterior solo se puede llevar a cabo si inicialmente hay una constante y eficaz comunicación entre familia- escuela, de manera que el proceso de enseñanza aprendizaje encuentre apoyo en cada una de estas instituciones con la finalidad de formar cuidadnos críticos, activos y propositivos capaces de tomar decisiones que puedan alimentar sus proyectos de vida de la manera más asertiva.

Es así como cada una de las instituciones aporta al desarrollo social y cultural de los estudiantes que a la vez se están formando como ciudadanos con capacidades que involucran "la identidad, los derechos y 
deberes, el sentido de la vida, el cuerpo y la naturaleza y finalmente la participación y convivencia"

Así que la intención de la escuela no puede ser satanizar todo aquello que los medios nos ofrecen, sino tener la capacidad de orientar hacia la televidencia crítica, reflexiva y sobre todo la importancia que tienen el poder reapropiar ideas que para el caso de la campaña audiovisual sobre prevención de consumo de sustancias psicoactivas es un ejercicio que repercute en la toma de decisiones en cuanto a la entrada y salida del universo SPA.

Finalmente, se invita a las instituciones en cuestión a reflexionar acerca de la formación que se está llevando a cabo en lo que respecta a la recepción, y cómo se puede optimizar dicho proceso mediante la contextualización de los contenidos televisivos y la crítica a los mismos en un acompañamiento continuo a los estudiantes.

Surge la necesidad de analizar la disposición que tiene el docente para aportar al campo de la Comunicación Educativa en el aula, así que a continuación se pondrán a consideración algunos retos para hacer la profesión docente una profesión transformadora.

\section{Docente - Comunicación Educativa}

Detrás de la idea de formar televidentes activos, críticos y propositivos, está la figura valiosa del docente, ¿somos realmente docentes formadores en comunicación?... realmente estamos apenas iniciando el arduo proceso de auto reconocimiento, pues a diario nos encontramos con situaciones desde las más simples hasta las más complejas relacionadas con la comunicación y sus implicaciones ambiciosas del campo de la educación.
A diario se generan discusiones a fin de encontrar un "perfil ideal" de docente que dé respuesta a un modelo que la sociedad espera ver y seguir, en medio de esta disyuntiva se vislumbra la verdadera misión del educador que es planteada desde diferentes perspectivas y exige sobrellevar un sinnúmero de retos.

Frente a este planteamiento Freire, hace referencia a ser portadores de una "Fuerza transformadora" que debe ir en contra de la ideología de la opresión (absolutización de la ignorancia) y a favor de una educación liberadora, una conciliación de polos. Para ello es necesario partir de un diálogo Educador-educando, se debe dar una comunicación efectiva, verdadera, comprensiva, pues de esta forma se llaga al conocimiento inicial de los sujetos que participan en el proceso de enseñanza/ aprendizaje.

Así que el campo de la Comunicación Educativa es un mar inmenso dispuesto en el océano del conocimiento, que posibilita diversos navegantes ansiosos de sabiduría, donde cada capitán lleva su tripulación con una responsabilidad compleja. El docente como capitán debe estar en constante diálogo de saberes para poder propiciar momentos significantes para sus estudiantes.

Lo anterior se logra tomando referentes del contexto próximo (escuela, familia, barrio, ciudad...) y todo aquello que es relevante "criticar" para comprender y proponer posibles soluciones a diversas problemáticas que se pueden llegar a dar. 


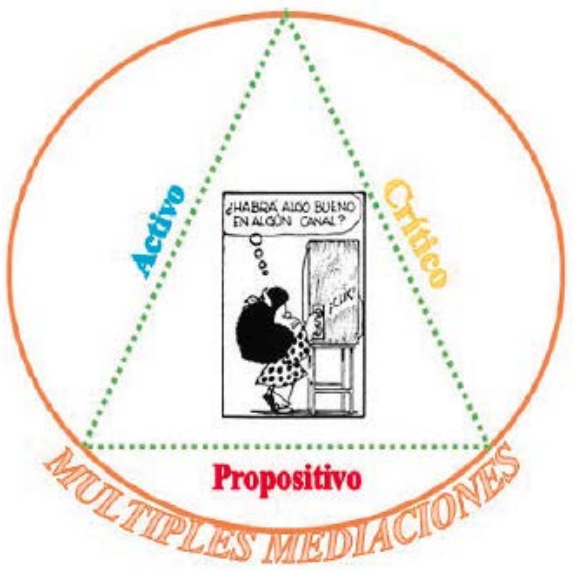

En el caso puntual de la recepción televisiva, la labor docente se torna complicada en primera instancia, debido a dos aspectos transcendentales, por un lado, la satanización constante que hacemos de los medios, de otro lado el tiempo excesivo que dedican nuestros estudiantes al acto de estar frente al televisor.

Es precisamente en esos dos momentos circunstanciales donde se dan las múltiples mediaciones, las cuales, en la medida que seamos capaces de identificar podemos llegar a hacer eco significativo en la orientación y formación de televidentes activos, críticos y propositivos. Esto constituye un triángulo ambicioso que toma al sujeto como referente de análisis constante.

Es durante el proceso de recepción cuando se interviene teniendo claridad en lo referido a las mediaciones que se dan en el acto televisivo, con el propósito de hacer de la televisión una herramienta pedagógica valiosa, y posibilitar la educación en medios.

La línea de salida está trazada, el reto es iniciar la transformación de manera intrínseca, resistiendo a cada ola en el mar de la comunicación para así proyectar una educación transformadora basada en la
Comunicación Educativa como vehículo ideal que permita la compresión de mundos posibles.

\section{Conclusiones}

Al realizar el análisis/síntesis y la discusión correspondiente, se pueden construir las siguientes conclusiones:

En el proceso de recepción ejercido por los estudiantes de grado $5^{\circ} \mathrm{JT}$ de la institución educativa Manuela Ayala de Gaitán, se visualiza la interacción como una de las micromediaciones fuertes que contienen a la vez el contexto, el ayudar y la comprensión (esto haciendo referencia a que la campaña audiovisual respondía a la prevención de consumo de sustancias psicoactivas), se puede extraer de aquí la relevancia que tienen para los menores participantes en dicha investigación el hecho de hacer parte de un contexto donde el consumo de sustancias psicoactivas es cada vez más cercano.

Se hacen explicitas las televidencias de primer orden, desde lo emocional a lo contextual. Los estudiantes/audiencias, resaltan la idea de contexto como aspecto influyente en la toma de decisiones para comportarse de una u otra manera; lo anterior parte de una individualización de los sujetos, debido a que cada estudiante vive en entornos distintos y con un grupo familiar particular, y se observan diferentes programas de televisión.

Se reflejan macromediaciones construidas a partir de las micromediaciones: televidencias de primer y segundo orden, teniendo en cuenta que las macromediaciones como lo explica Orozco, transcurren como un proceso múltiple: no hay televidencias puras porque existen otras mediaciones diferentes a las individuales, situacionales y contextuales que conforman la televidencia y que se manifiestan en 
diversos escenarios: identidad, percepción, instituciones, familia.

Los estudiantes en su condición de audiencias tienen bastante claras las funciones de las instituciones y dejan ver una preocupación grupal frente a la función de la familia, la televisión y la escuela en la prevención del consumo de sustancias psicoactivas; aunque parezca que los estudiantes/audiencias no muestran preocupación por la prevención de consumo, debido a la idea que se tiene respecto a los comportamientos de los adolescentes que hoy día viven en función de experimentar y probar lo que pase por su vista, aquí se evidencia una necesidad inicial de estar informados en lo que se refiere a causas y consecuencias del consumo.

Las mediaciones compuestas por familiaescuela, atan a los receptores a una serie de condiciones sociales e históricas concretas, las cuales no solo hacen complejo el fenómeno, sino también incompatible, "pues entre menos impacto tengan la institución familiar y escolar en los menores, mayor será la importancia de la televisión en sus dinámicas, pero también al contrario: a mayor mediación familiar y escolar, menor efecto en la producción de significados que devienen de los medios" (Orozco, 2001: 63).

La responsabilidad en la formación de nuestros estudiantes es compartida por las instituciones familia- escuela, puesto que el estudiante como televidente inicia un proceso de resistencia (mediaciones), frente a la campaña audiovisual sobre prevención de consumo de sustancias psicoactivas, a la vez se dan unas interacciones entre lo que ve y vive; paralelamente, el estudiante propone y espera una respuesta de dichas instituciones en lo que se refiere a prevención y mitigación de la problemática de consumo.
Es posible reconocer mediaciones (macro y micro) en el proceso de recepción de la "campaña antidrogas 2011", debido a que los estudiantes/audiencias, se incorporan en una secuencia interactiva que implica diversos grados de involucramiento y procesamiento del contenido televisivo, lo cual repercute en la reapropiación de los mismos contenidos.

El docente, desde la comunicación educativa, tiene la función de acompañar el proceso de recepción dado en los actos televisivos, con el propósito de hacer de la televisión una herramienta pedagógica y posibilitar la educación en medios.

La escuela está en la capacidad de orientar hacia la televidencia crítica, reflexiva y sobre todo la importancia que tienen el poder reapropiar ideas que para el caso de la campaña audiovisual sobre prevención de consumo de sustancias psicoactivas es un ejercicio que repercute en la toma de decisiones en cuanto a la entrada y salida del universo SPA. Para ello es importante crear espacios de disertación alrededor de las problemáticas que aquejan a la comunidad educativa.

La presente propuesta de investigación permitió reconocer la creciente importancia de la televisión dentro de los hogares, por ello dicha institución se ha transformado en uno de los principales objetos de estudio de diversas disciplinas. Si bien su actuación entra en convergencia con otros medios de similar importancia como internet, es posible decir que hoy en día la televisión es considerada uno de los medios de comunicación más populares y con mayor influencia en todos los estratos de las sociedades. Así que es necesario que a nivel central de la secretaria Distrital de educación (Bogotá), se propicien espacios de orientación dirigida a la comunidad educativa (estudiantes, padres de familia 
y docentes) respecto a la influencia que tiene la televisión y las mediaciones que se pueden llegar a dar, de esta manera la televisión en el aula podría ser una de las herramientas más transformadoras en beneficio de los estudiantes.

\section{Referencia Bibliográfica}

Arroyo, I., Baños, M. \& Wyck V. (2013). "Análisis de los mensajes audiovisuales del Tercer Sector en YouTube". En: Revista Latina de Comunicación Social $N^{\circ}$ 068, 328 -354. Consultado el 2 de febrero de 2010, http:// www.revistalatinacs.org/068/paper/980 Madrid/14_Arroyo.html

Bustamante, E. (abril de 2000). Las políticas públicas en la Unión Europea. En: V Encuentro de Ciencias de la Comunicación (ALAIC) en Santiago de Chile.

Camarero, E. \& Marcos, M. (2012). “Campañas en televisión contra la violencia de Género del ministerio de sanidad, política social e Igualdad (2008-2011). Análisis de contenidos previo al Estudio de recepción". Comunicación Vivat Academia $\mathrm{N}^{\circ} 121,17-30$. Consultado el 8 de Febrero de 2013, http://www.vivatacademia. net/index.php/vivat/article/view/16

Chicharro, M. (2011). "Aprendiendo de la ficción televisiva. La recepción y los efectos socializadores de "Amar en tiempos revueltos"”. Comunicar, $\mathrm{N}^{\circ} 36,181-189$. Consultado el 8 de Febrero de 2013,http://www.revistacomunicar. com/index.php? contenido $=$ detalles\&numero $=$ $36 \&$ articulo $=36-2011-22$

Del Valle, C., Denegri M., \& Chávez, D. (2012). "Alfabetización audiovisual y consumo de medios y publicidad en universitarios de Pedagogía en Chile". Comunicar, $\mathrm{N}^{\circ}$ 38, 183-191. Consultado el 4 de Febrero de 2013, http://dialnet.unirioja.es/descarga/ articulo/3851506/2.pdf.

Fuenzalida, V. (2011). "Resignificar la educación televisiva: desde la escuela a la vida cotidiana". Comunicar, $\mathrm{N}^{\circ} 36,15-24$. Consultado el 1 de Febrero de 2012, http://www. revistacomunicar.com/index.php? contenido $=\mathrm{d}$ etalles\&numero $=36 \&$ articulo $=36-2011-03$
García, E. A. (2009). "Análisis de la competencia televisiva en la interacción maestro - estudiante de básica secundaria y su impacto en la audiencia adolescente televisiva. Universidad Tecnológica de Pereira. Pereira, Colombia". Consultado el 1 de Febrero de 2012,http://repositorio.utp.edu.co/dspace/ handle/11059/1517

Jensen, K. (1987) "Qualitative Audience Research: Towards an Integrative Approach to Reception". Critical studies in mass communication 4, 21-36.

Medina, A \& Méndiz, A. (2012). "La representación de la infancia en la publicidad: Panorama por el marco deontológico español." Pensar la Publicidad, Vol. 6, $\mathrm{N}^{\mathrm{o}}$ 1, 231-253. Concultado el 18 de Febrero de 2012, http://dialnet.unirioja.es/servlet/ articulo? codigo $=4030629$.

Moreno, J. A., Rosa, A., López, A., y Pérez, B. C. (2007). La representación gráfica como recurso educativo en la enseñanza de las actividades acuáticas. NSW, XXIX (1), 39-45.

Muñoz, g. \& Judikis, J. (2012). "Prácticas de recepción televisiva en estudiantes de enseñanza media de Punta Arenas." Magallania, Vol 40, 127-136. Consultado el 17 de Febrero de 2012, http://www.scielo.cl/scielo.php?script=sci arttext\&pid=S0718-22442012000100007

Orozco, Guillermo (1987). Televisión y producción de significados (Tres ensayos). México: Universidad Iberoamericana.

G. (2001). "Audiencias, televisión y educación: una deconstrucción pedagógica de la televidencia y sus mediaciones." En: Revista Iberoamérica de Educación $\mathrm{N}^{\circ} 27$. Publicado por la Organización de Estudios Iberoamericanos, pp. 155-175. Consultado el 25 de Febrero de 2012, http://www.rieoei.org/ rie27.htm.

G. (2001) "Televidencias y Mediaciones" (Capítulo 1). En: Televisión, Audiencias y Educación, Editorial Norma. (pp. 23).

G. 2012. Televisión y producción de interacciones comunicativas. En: Revista Nueva época. 39- 54. 
Ortiz, M., Ruiz J. \& Díaz, E. (2012). “¿Están las cadenas de televisión interesadas en una mejor TV para los menores? Las televisiones y la investigación en infancia y televisión." Comunicar, $\mathrm{N}^{\mathrm{o}} 40,137-144$. Consultado el 25 de Febrero de 2012, www.revistacomunicar. com.

República de Colombia. Consejo de Bogotá. (2012). Plan de desarrollo: un acuerdo para la ciudad: Política educativa en la Bogotá humana, 2012-2016. Plan de desarrollo: un acuerdo para la ciudad. Bogotá D.C.

Reyes, T. (2000). "Métodos cualitativos de investigación: los grupos focales $\mathrm{y}$ el estudio de caso". Consultado el 7 de Febrero de 2012, http:// www.fvet.uba.ar / rectorado/postgrado/especialidad/programas/ Grupofocalyestudiodecaso.pdf.

Rincón, O. (2001). Televisión, video y subjetividad. Bogotá: Norma.

Valdettaro, S. (2011). El dispositivo - Mc Luhan. Recuperaciones y derivaciones. Argentina: Universidad Nacional del Rosario.

Winocur, R. (2009). Robinsoe Crusoe ya tiene celular. México: Siglo

\section{Notas al lector}

1 República de Colombia. . Consejo de Bogotá. (2012). Plan de desarrollo: un acuerdo para la ciudad: Política educativa en la Bogotá humana, 2012-2016. Plan de desarrollo: un acuerdo para la ciudad. Bogotá D.C. 